\title{
Hadron resonance gas with repulsive mean field interaction: Thermodynamics and transport properties
}

\author{
Guruprakash Kadam $\circledast^{1, *}$ and Hiranmaya Mishra ${ }^{2, \dagger}$ \\ ${ }^{1}$ Department of Physics, Shivaji University, Kolhapur, Maharashtra-416004, India \\ ${ }^{2}$ Theory Division, Physical Research Laboratory, Navarangpura, Ahmedabad-380 009, India
}

(Received 8 July 2019; published 14 October 2019)

\begin{abstract}
We discuss the interacting hadron resonance gas model to describe the thermodynamics of hadronic matter. While the attractive interaction between hadrons is taken care of by including all the resonances with zero width, the repulsive interactions between them are described by a density-dependent mean field potential. The bulk thermodynamic quantities are confronted with the lattice quantum chromodynamics simulation results at zero as well as at finite baryon chemical potential. We further estimate the shear and bulk viscosity coefficients of hot and dense hadronic matter within the ambit of this interacting hadron resonance gas model.
\end{abstract}

DOI: 10.1103/PhysRevD.100.074015

\section{INTRODUCTION}

Understanding the phase diagram of strongly interacting matter is one of the important and challenging topics of current research in strong interaction physics-both theoretically and experimentally. The theoretical framework describing nuclear matter at a fundamental level is quantum chromodynamics (QCD). At low temperature $(T)$ and low baryon chemical potential $(\mu)$ the fundamental degrees of freedom of QCD are colorless hadrons, while at high temperature and high baryon density the fundamental degrees of freedom are colored quarks and gluons. Lattice quantum chromodynamics (LQCD) simulations at zero chemical potential and finite temperature suggest a crossover transition for QCD matter from a hadronic phase to a quarkgluon-plasma (QGP) phase [1-6]. At zero chemical potential, the chiral crossover temperature is estimated to be $T_{c} \sim 156 \mathrm{MeV}$ [7]. While LQCD simulations at vanishing chemical potential have been quite successful, LQCD simulations at finite $\mu$ have been rather challenging, particularly at high $\mu$, leading to large uncertainties in estimating the transition line in the $T-\mu$ plane of the QCD phase diagram [8]. At small $\mu$, however, precise computation of the transition line has been carried out recently [9-11].

The low energy effective models of QCD, viz., the Nambu-Jona-Lasinio model [12,13], the quark-mesoncoupling model [14], etc., provide a reasonable theoretical

\footnotetext{
*guruprasadkadam18@gmail.com

hm@prl.res.in
}

Published by the American Physical Society under the terms of the Creative Commons Attribution 4.0 International license. Further distribution of this work must maintain attribution to the author(s) and the published article's title, journal citation, and DOI. Funded by SCOAP. framework to explore strongly interacting matter below the QCD transition temperature, $T_{c}$. These models are based on certain QCD symmetries, and they are tremendously successful in describing many features of the QCD phase diagram at zero as well at finite baryon density. Apart from these symmetry-based models, another model that has also been tremendously successful in describing the low temperature hadronic phase of QCD is the hadron resonance gas model (HRG). The hadron resonance gas model is the statistical model of QCD describing the low temperature hadronic phase of quantum chromodynamics. This model is based on the so-called Dashen-Ma-Bernstein theorem, which allows us to compute the partition function of the interacting system of hadrons in terms of the scattering matrix [15]. Using this S-matrix formulation of statistical mechanics, it can be shown that if the dynamics of thermodynamic system of hadrons is dominated by narrow-resonance formation, then the resulting system essentially behaves like a noninteracting system of hadrons and resonances [16-18]. This ideal HRG model, despite its success in describing hadron multiplicities in heavy-ion collisions [19-27], fails to account for the short-range repulsive interactions between hadrons. It has been shown that the repulsive interactions modeled via excluded volume can have a significant effect on thermodynamic observables, especially higher order fluctuations [28-31], as well as in the context of statistical hadronization [32]. One possible way to include these repulsive interactions is through the van der Waals excluded volume procedure $[33,34]$. Another approach is to treat the repulsive interactions in a mean field way $[35,36]$. Recently, the relativistic mean field approach has been used to calculate the fluctuations of conserved charges [37]. This work discussed the repulsive mean field interactions which are 
present only at finite baryon density. They showed that the deviations of higher order fluctuations estimated using the ideal HRG can be accounted for by means of repulsive interactions treated in a mean field way. The failure of the ideal HRG model to explain the thermodynamical observable can be attributed to the fact that at high temperature and density, the relativistic virial expansion up to the second-order virial coefficient cannot be a reasonable approximation, and the validity of the HRG model needs to be checked against its agreement with LQCD results.

In the past few decades relativistic and ultrarelativistic heavy-ion collision experiments have provided a unique opportunity to study the phase diagram of QCD. The relativistic hydrodynamics has been tremendously successful in simulating the evolution of matter created in HIC experiments [38-46]. In the relativistic hydrodynamic simulations the coefficients of shear and bulk viscosities influence various observables, viz., the flow coefficients and the transverse momentum distribution of produced particles. In fact, it has been found that a finite but very small shear viscosity-to-entropy ratio should be included in the hydrodynamic description to explain elliptic flow data $[47,48]$. Further, $\eta / s$ obtained using AdS/CFT correspondence [49] has put the lower bound on its value equal to $\frac{1}{4 \pi}$, called the Kovtun-Son-Starinets (KSS) bound. This interesting finding has motivated many theoretical investigations to understand and rigorously derive this ratio from a microscopic theory [50-64]. The bulk viscosity coefficient $(\zeta)$ is also important to include in the dissipative hydrodynamics. During the expansion of the fireball, when the temperature approaches the critical temperature, $\zeta$ can be large and can give rise to different interesting phenomena like cavitation when the pressure vanishes and the hydrodynamic description breaks down $[65,66]$. The effect of bulk viscosity on the particle spectra and flow coefficients has been investigated in [67-69], while the interplay of shear and bulk viscosity coefficients has been studied in Refs. [70-72]. The coefficient of bulk viscosity has been estimated for both the hadronic and the partonic systems [73-107].

Hydrodynamic simulation of matter created in HIC collision requires information regarding the equation of state (EOS) as well as the transport coefficients. In this work we analyze the QCD equation of state of hadronic matter at finite baryon chemical potential. We employ the hadron resonance gas model to estimate all the thermodynamic quantities. While the attractive interactions between hadrons are accounted for by including all the resonance states up to $2.25 \mathrm{GeV}$, the short-range repulsive interactions among hadrons are treated in the mean field approach. We call this model the relativistic mean field hadron resonance gas model (RMFHRG). The RMFHRG differs from the Walecka-type mean field models in the sense that in the former, the repulsive mean field interactions are present even at zero baryon density unlike in the latter case. We also estimate the shear and bulk viscosity coefficients of hadronic matter within the ambit of the RMFHRG.
We organize the paper as follows. In Sec. II we compute the pressure and other bulk thermodynamic quantities for the interacting hadron resonance gas with a repulsive mean field interaction. In Sec. III, we discuss the results for the thermodynamics and confront them with the lattice simulation results both at zero and finite chemical potential. We then estimate the viscosity coefficients for hot and dense hadronic matter within the ambit of the HRG model with mean field interactions. Finally, in Sec. IV, we summarize the findings of the present investigation and give a possible outlook.

\section{HADRON RESONANCE GAS MODEL WITH A REPULSIVE MEAN FIELD POTENTIAL}

Thermodynamic properties of the hadron resonance gas model can be deduced from the grand canonical partition function defined as

$$
\begin{aligned}
\mathcal{Z}(V, T, \mu)= & \int d m\left[\rho_{b}(m) \ln Z_{b}(m, V, T, \mu)\right. \\
& \left.+\rho_{f}(m) \ln Z_{f}(m, V, T, \mu)\right]
\end{aligned}
$$

where $\rho_{b}$ and $\rho_{f}$ are the mass spectrum of the bosons and fermions, respectively. We assume that the hadron mass spectrum is given by

$$
\rho(m)=\sum_{a}^{\Lambda} g_{a} \delta\left(m-m_{a}\right) \theta(\Lambda-m)
$$

where $g_{a}$ is the degeneracy and $m_{a}$ is the mass of the $a$ th hadronic species. This discrete mass spectrum consists of all the experimentally known hadrons with cutoff $\Lambda$. One can set different cutoff values for baryons and mesons.

To include the effect of a repulsive interaction among hadrons, we use a repulsive mean field approach as was used in Refs. [35,36] and more recently in the case of baryons in Ref. [37]. In this approach, it is assumed that the repulsive interactions lead to a shift in the single particle energy and is given by

$$
\varepsilon_{a}=\sqrt{p^{2}+m_{a}^{2}}+U(n)=E_{a}+U(n)
$$

where $E_{a}=\sqrt{\mathbf{p}^{2}+m_{a}^{2}}$ and $n$ is the total hadron number density. The potential energy $U$ represents a repulsive interaction between hadrons, and it is taken to be a function of total hadron density $n$. For any arbitrary interhadron potential $V(\mathbf{r})$, the potential energy is $U(n)=K n$. Here, the phenomenological parameter $K$ is given by the integral of the potential $V(\mathbf{r})$ over the spatial volume $[35,36]$.

In this work, we assign different repulsive interaction parameters for baryons and mesons. We denote the mean field parameter for baryons $(B)$ and antibaryons $(\bar{B})$ by $K_{B}$, 
while for mesons we denote it by $K_{M}$. Thus, for baryons (antibaryons)

$$
U\left(n_{B\{\bar{B}\}}\right)=K_{B} n_{B\{\bar{B}\}}
$$

and for mesons

$$
U\left(n_{M}\right)=K_{M} n_{M}
$$

The total hadron number density is

$$
n(T, \mu)=\sum_{a} n_{a}=n_{B}+n_{\bar{B}}+n_{M}
$$

where $n_{a}$ is the number density of the $a$ th hadronic species. Note that $n_{B}, n_{\bar{B}}$, and $n_{M}$ are total baryon, antibaryon, and meson number densities, respectively. Explicitly, for baryons,

$$
n_{B}=\sum_{a \in B} \int d \Gamma_{a} \frac{1}{e^{\left(\frac{E_{a}-\mu_{\text {eff }}^{a}}{T}\right)}+1}
$$

where the sum is over all the baryons. Note that $d \Gamma_{a} \equiv \frac{g_{a} d^{3} p}{(2 \pi)^{3}}$, and $\mu_{\text {eff }}^{a}=q^{a} \mu-U\left(n_{B}\right)$ is a baryon effective chemical potential, with $q^{a}$ being the baryonic charge of the $a$ th baryon and $\mu$ the baryon chemical potential. Similarly, for antibaryons

$$
n_{\bar{B}}=\sum_{a \in \bar{B}} \int d \Gamma_{a} \frac{1}{e^{\left.\frac{E_{a}-\bar{\mu}_{\text {eff }}^{a}}{T}\right)}+1}
$$

where $\bar{\mu}_{\text {eff }}^{a}=\left(\bar{q}^{a} \mu-U\left(n_{\bar{B}}\right)\right)$ is an antibaryon effective chemical potential with $\bar{q}^{a}=-q^{a}$, which is the corresponding baryonic charge. For mesons,

$$
n_{M}=\sum_{a \in M} \int d \Gamma_{a} \frac{1}{e^{\frac{\left(E_{a}-K_{M} n_{M}\right)}{T}}-1}
$$

where the sum is over all the mesons. Note that $\mu=0$ for mesons since the baryon charge is zero for them. In the Boltzmann approximation, momentum integration can be readily performed, and one can obtain much simpler expressions for the number density. For baryons we get

$$
\begin{aligned}
& n_{B}=\sum_{a \in B} \frac{g_{a}}{2 \pi^{2}} m_{a}^{2} T \mathcal{K}_{2}\left(\frac{m_{a}}{T}\right) e^{\frac{\mu_{\text {eff }}^{a}}{T}}, \\
& n_{\bar{B}}=\sum_{a \in \bar{B}} \frac{g_{a}}{2 \pi^{2}} m_{a}^{2} T \mathcal{K}_{2}\left(\frac{m_{a}}{T}\right) e^{\frac{\bar{\mu}_{\text {eff }}^{a}}{T}},
\end{aligned}
$$

where $\mathcal{K}_{n}(z)$ is the modified Bessel function of order $n$. For mesons we get

$$
n_{M}=\sum_{a \in M} \frac{g_{a}}{2 \pi^{2}} m_{a}^{2} T \mathcal{K}_{2}\left(\frac{m_{a}}{T}\right) e^{-\frac{K_{M} n_{M}}{T}}
$$

Equations (10)-(12) are self-consistent equations for number density which can be solved numerically.

The total baryon (antibaryon) energy density is

$\epsilon_{B\{\bar{B}\}}=\sum_{a \in B\{\bar{B}\}} \int d \Gamma_{a} \frac{\varepsilon_{a}}{e^{\frac{\left.\left[E_{a}-\mu_{\mathrm{eff}}^{a} \bar{\mu}_{\mathrm{eff}}\right\}\right]}{T}}+1}+\phi_{B\{\bar{B}\}}\left(n_{B\{\bar{B}\}}\right)$,

and for mesons

$$
\epsilon_{M}=\sum_{a \in M} \int d \Gamma_{a} \frac{\varepsilon_{a}}{e^{\frac{\varepsilon_{a}}{T}}-1}+\phi_{M}\left(n_{M}\right)
$$

where $\phi(n)$ represents the correction to the energy density in order to avoid double counting the potential. It can be determined using the condition that $\varepsilon_{a}=\frac{\partial \epsilon}{\partial n_{a}}$. Taking the derivative of the baryon energy density and using Eq. (4), we get

$$
\frac{\partial \phi_{B\{\bar{B}\}}}{\partial n_{B\{\bar{B}\}}}=-K_{B} n_{B\{\bar{B}\}}
$$

and hence

$$
\phi_{B}\left(n_{B\{\bar{B}\}}\right)=-\frac{1}{2} K_{B} n_{B\{\bar{B}\}}^{2} .
$$

Similarly, for mesons one can obtain

$$
\phi_{M}\left(n_{M}\right)=-\frac{1}{2} K_{M} n_{M}^{2}
$$

Pressure of the gas can now be readily obtained. For baryons

$$
\begin{aligned}
P_{B\{\bar{B}\}}(T, \mu)= & T \sum_{a \in B\{\bar{B}\}} \int d \Gamma_{a} \ln \left[1+e^{\left.-\left(\frac{E_{a}-\mu_{\text {eff }}^{a}\left\{\tilde{\mu}_{\text {eff }}^{a}\right\}}{T}\right)\right]}\right. \\
& -\phi_{B\{\bar{B}\}}\left(n_{B\{\bar{B}\}}\right)
\end{aligned}
$$

and for mesons

$$
P_{M}(T)=T \sum_{a \in M} \int d \Gamma_{a} \ln \left[1+e^{-\left(\frac{\varepsilon_{a}}{T}\right)}\right]-\phi_{M}\left(n_{M}\right) .
$$

Finally, entropy density can be obtained from the fundamental thermodynamic relation $s=(\epsilon+P-\mu n) / T$. It is worth noting that the effective interaction model we are considering is different from the relativistic Lagrangian model. In the latter case the repulsive mean fields are present only at nonzero baryon density, while in the former 
case the repulsive interactions are present even at zero baryon density.

\section{RESULTS AND DISCUSSION}

In the hadron resonance gas model it is customary to include all the hadrons and resonances up to a certain cutoff $\Lambda$. We include all the mesons and baryons up to $\Lambda=2.25 \mathrm{GeV}$ listed in the Ref. [108]. The phenomenological parameter $K$ is the spatially integrated value of the interhadron repulsive potential. In Ref. [35] the potential was taken to be the same for all hadrons, i.e., for all the baryons and the mesons. In the present work we have taken this parameter different for mesons and baryons. For baryons we have taken it to be the same for all the baryons, and the value is taken as in Ref. [37], namely, $K_{B}=$ $450 \mathrm{MeV} \mathrm{fm}^{3}$ for all baryons. Although different lattices as well as chiral effective theories indicate that the strength can be different for nucleon-nucleon, hyperon-hyperon, or nucleon-hyperon interactions, there is not enough information about hadrons to have a more realistic and sophisticated mean field model. For the mesons we have taken a much smaller value for the repulsion parameter $K_{M}=$ $50 \mathrm{MeV} \mathrm{fm}^{3}$ in the present study. The motivation in choosing these two phenomenological parameters has been that the lattice results are reasonably reproduced regarding thermodynamics, and then use these parameters to estimate the viscosity parameters, both at zero and at finite density.

Figure 1 shows the scaled pressure and the interaction measure estimated within the ambit of the RMFHRG (blue solid curve) at vanishing baryon chemical potential. The dashed curve corresponds to ideal HRG results, while the circles with error bars correspond to lattice QCD simulation results [8]. We note that the effect of including the repulsive

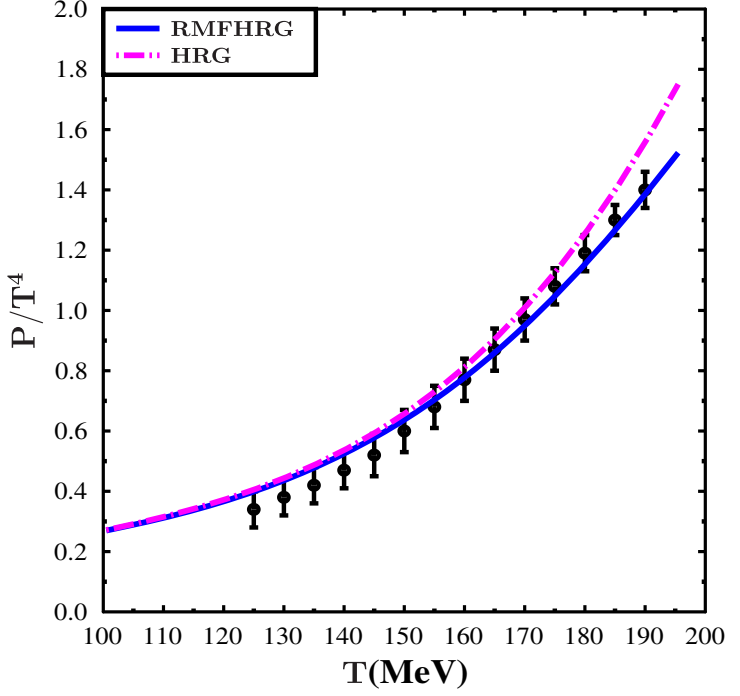

(a) mean field interaction is to suppress the thermodynamical quantities compared to their ideal HRG estimation counterpart (dashed magenta curve). While the HRG pressure [Fig. 2(a)] starts to deviate from the lattice results at $T \sim 160 \mathrm{MeV}$, the RMFHRG estimation agrees with the lattice results all the way up to $190 \mathrm{MeV}$. It is not reasonable to push the HRG model results above the QCD transition temperature $\left(T_{c}\right)$, which LQCD estimates to lie in the range $155-160 \mathrm{MeV}$ [7]. The reason for this is twofold. First, the HRG approximation of the hadronic matter might break down at high density near and above $T_{c}$. Second, the hadrons do not exist above $T_{c}$. But a recent study [29] shows that the hadrons do not melt quickly as one would expect on the basis of the ideal HRG model. In this study the authors analyzed the possible improvement of the ideal hadron resonance gas model by including the repulsive interactions between baryons. If one includes the attractive and repulsive interactions between the baryons through van der Waals parameters, while keeping the meson gas ideal, the pressure of the hadron gas agrees with the LQCD data all the way above $T_{c}$. We may similarly conclude that the inclusion of repulsive mean fields might push the validity of the HRG model well above $T_{c}$. Nonetheless, we do not have any other strong reason to believe this except for the apparent agreement with the LQCD results.

Unlike pressure the interaction measure is somewhat below LQCD results above $T=150 \mathrm{MeV}$. It is an established fact that the so-called heavy Hagedorn states, which are missing in our model, contribute significantly to the energy density. The rapid rise of energy density can be explained by extending the ideal HRG model by including continuum Hagedorn states along with the discrete states above the cut off $\Lambda$ in the density of states [78].

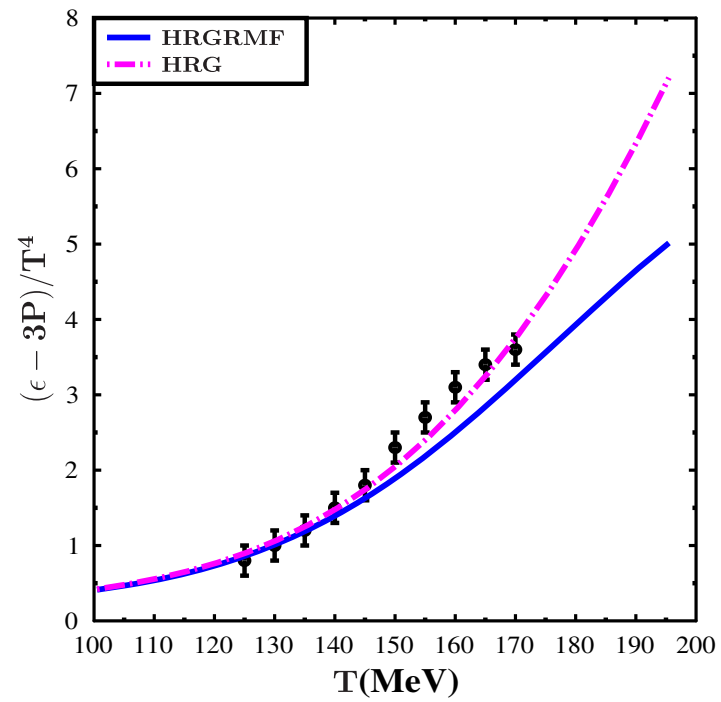

(b)

FIG. 1. Scaled pressure (left panel) and the interaction measure (right panel) in the RMFHRG and ideal HRG for $\mu=0$. The lattice data are taken from Ref. [8]. 


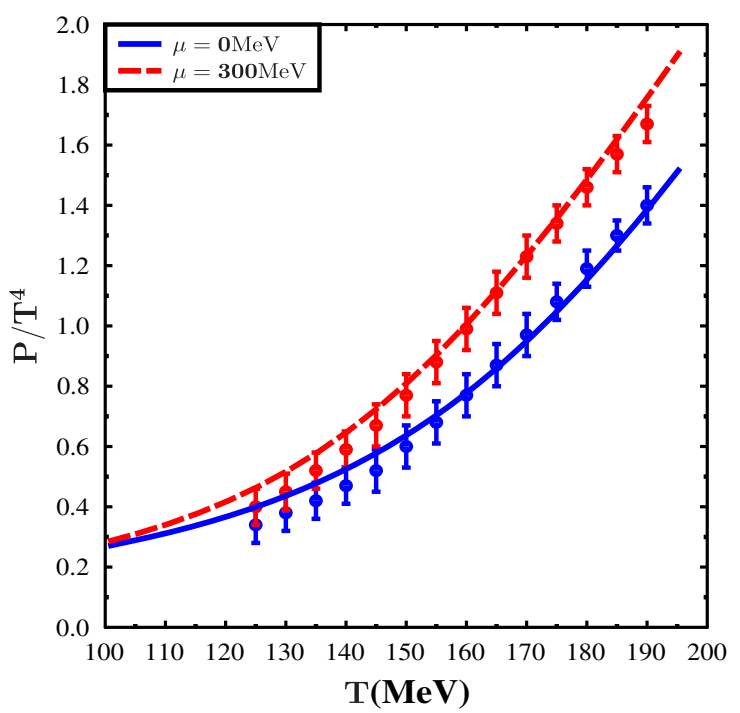

(a)

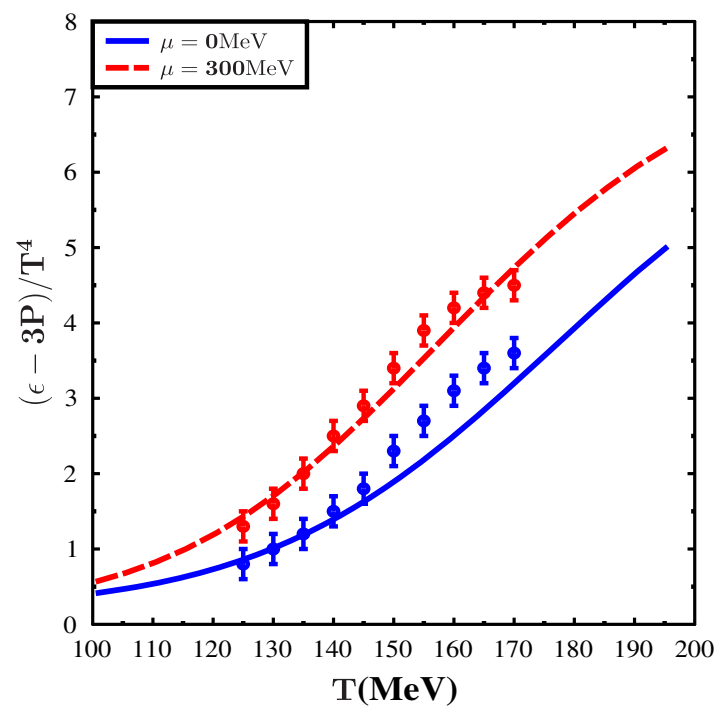

(b)

FIG. 2. Scaled pressure (left panel) and the interaction measure (right panel) in the RMFHRG model at finite baryon chemical potential. The lattice data are taken from Ref. [8].

Figure 2 shows the scaled pressure and interaction measure at finite baryon chemical potentials estimated within the ambit of the RMFHRG. We note that the RMFHRG is in reasonable agreement with LQCD results even at finite baryon density. Further, the interaction measure is in better agreement with the LQCD results at finite density than in the $\mu=0$ case. However, while making this observation, we have to keep in mind that the lattice data of Ref. [8] are estimated at order $\mu^{2}$. Figure 3 shows the adiabatic speed of sound at finite baryon density. The RMFHRG estimations of $C_{s}^{2}$ are within the error bars of

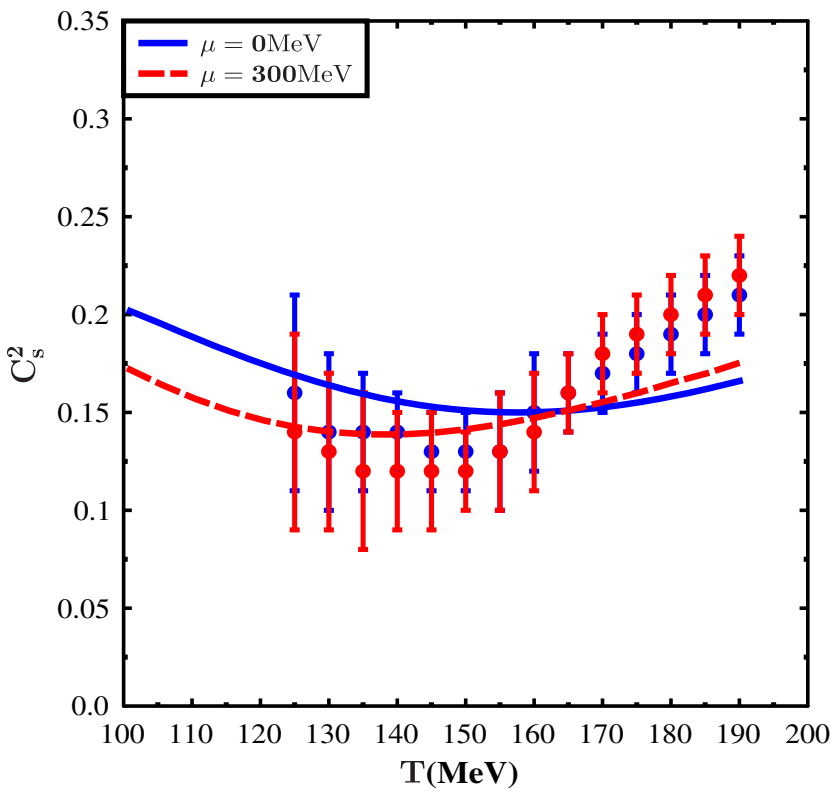

FIG. 3. Speed of sound in the RMFHRG model at finite baryon density. The lattice data are taken from Ref. [8]. the LQCD results. Further, the $C_{s}^{2}$ has a minimum at $T=$ $155 \mathrm{MeV}$ for $\mu=0$ and at $T=140 \mathrm{MeV}$ for $\mu=300 \mathrm{MeV}$, which is in very close agreement with the LQCD results.

The coefficients of shear and bulk viscosities can be extracted from the relativistic Boltzmann equation. These have been derived in Refs. [50,51] in the absence of any mean fields. While various authors have used different types of mean fields to include medium effects as well as interactions and have derived the transport coefficients, a rigorous, thermodynamically consistent derivation for the expressions for different transport coefficients was derived in Ref. [109], both in the presence of a scalar and a vector mean field. The scalar mean field affects the mass, while the repulsive vector mean field affects the chemical potential. The potential considered here does not affect the masses of the hadrons, and it is like a repulsive vector field; its effect is manifested in the effective chemical potential. In the relaxation time approximation of the Boltzmann equation, the shear $(\eta)$ and bulk viscosity $(\zeta)$ coefficients are given by [50,51,109]

$$
\begin{gathered}
\eta=\frac{1}{15 T} \sum_{a} \int \frac{d^{3} p}{(2 \pi)^{3}} \frac{p^{4}}{E_{a}^{2}}\left(\tau_{a} f_{a}^{0}+\bar{\tau}_{a} \bar{f}_{a}^{0}\right) \\
\zeta=\frac{1}{T} \sum_{a} \int \frac{d^{3} p}{(2 \pi)^{3}}\left\{\tau_{a} f_{a}^{0}\left[E_{a} C_{n_{\mathcal{B}}}^{2}+\left(\frac{\partial P}{\partial n_{\mathcal{B}}}\right)_{\epsilon}-\frac{p^{2}}{3 E_{a}}\right]^{2}\right. \\
\left.+\bar{\tau}_{a} \bar{f}_{a}^{0}\left[E_{a} C_{n_{\mathcal{B}}}^{2}-\left(\frac{\partial P}{\partial n_{\mathcal{B}}}\right)_{\epsilon_{a}}-\frac{p^{2}}{3 E_{a}}\right]^{2}\right\}
\end{gathered}
$$

where $f^{0}$ is the equilibrium distribution function with an effective chemical potential including the mean field and $C_{n_{\mathcal{B}}}^{2}$ is the speed of sound at constant baryon number 
density. Further, in Eqs. (21) and (20), $\tau_{a}$ is the relaxation time for the $a$ th hadronic particle species, while the barred quantities correspond to those of antiparticles. In this work we use the thermally averaged relaxation time, which for a given species $a$ is given by

$$
\tau_{a}^{-1}=\sum_{b} n_{b}\left\langle\sigma_{a b} v_{a b}\right\rangle
$$

In the above, the sum is over all particles $(b)$ other than the particle $a$ with which the scattering takes place; $\sigma_{a b}$ is the total scattering cross section for the process $a\left(p_{a}\right)+b\left(p_{b}\right) \rightarrow c\left(p_{c}\right)+d\left(p_{d}\right)$, and $v_{a b}$ is the relative velocity given by

$$
v_{a b}=\frac{\sqrt{\left(p_{a} \cdot p_{b}\right)^{2}-m_{a}^{2} m_{b}^{2}}}{E_{a} E_{b}} .
$$

Further, $n_{b}$ is the number density for particle species $b$ given, with $g_{b}$ as the corresponding degeneracy factor, as

$$
n_{b}=\frac{g_{b}}{(2 \pi)^{3}} \int d \boldsymbol{p} f_{b}(\boldsymbol{p}) \simeq \frac{g_{b} T^{3}}{2 \pi^{2}}(\beta m)^{2} \mathcal{K}_{2}(\beta m) \exp \left(\beta \mu_{\text {eff }}^{b}\right)
$$

where the last step is written down in the Boltzmann approximation; here $\mu_{\text {eff }}^{b}=\mu-K_{B} n_{B}$ for baryons, $\mu_{\text {eff }}^{b}=$ $\bar{\mu}-K_{B} n_{\bar{B}}$ for antibaryons, and $\mu_{\text {eff }}^{b}=-K_{M} n_{M}$ for mesons.

Finally, the thermal average cross section $\left\langle\sigma_{a b} v_{a b}\right\rangle$ is given as

$$
\left\langle\sigma_{a b} v_{a b}\right\rangle=\frac{\int d^{3} p_{a} d^{3} p_{b} \sigma_{a b} v_{a b} f_{a}\left(p_{a}\right) f_{b}\left(p_{b}\right)}{\int d^{3} p_{a} d^{3} p_{b} f_{a}\left(p_{a}\right) f_{b}\left(p_{b}\right)} .
$$

The only unknown quantity in Eq. (25) is the total cross section. We estimate it as follows. In the Born approximation, the scattering amplitude $f(\theta, \phi)$ for a particle with mass $m$ that encounters a scattering potential $V(r)$ is given by [110]

$$
f(\theta, \phi)=-\frac{m}{2 \pi} \int d^{3} r V(r)=-\frac{m K}{(2 \pi)}
$$

and thus the cross section is given by

$$
\sigma=4 \pi\left(\frac{m K}{2 \pi}\right)^{2}
$$

Then the thermal averaged cross section can be written as $[111,112]$

$$
\begin{aligned}
\left\langle\sigma_{a b} v_{a b}\right\rangle= & \frac{\sigma}{8 m_{a}^{2} m_{b}^{2} \mathcal{K}_{2}\left(\beta m_{a}\right) \mathcal{K}_{2}\left(\beta m_{b}\right)} \\
& \times \int_{\left(m_{a}+m_{b}\right)^{2}}^{\infty} d S \frac{\left[S-\left(m_{a}-m_{b}\right)^{2}\right]}{\sqrt{S}} \\
& \times\left[S-\left(m_{a}+m_{b}\right)^{2}\right] \mathcal{K}_{1}(\beta \sqrt{S})
\end{aligned}
$$

where $\sqrt{S}$ is the center-of-mass energy. Clearly, we have suppressed the baryon/meson index in the expression for the cross section in the parameter $K$ in Eq. (27). It may be relevant here to mention that, while the cross section is independent of temperature and chemical potential, the thermal averaged cross section $\langle\sigma v\rangle$, in general, depends upon temperature and chemical potential. However, in the Boltzmann approximation $\langle\sigma v\rangle$ is independent of $\mu$. After evaluating the thermal averaged relaxation time for each species, we estimate the viscosity coefficients using Eqs. (20) and (21).

Figure 4 shows the ratio of shear viscosity to entropy density as a function of temperature. We have compared the ratio $\eta / s$ estimated within the ambit of the RMFHRG with various other model calculations $[49,58,76,95,96]$. The red dashed curve corresponds to the Chapman-Enscog method with constant cross sections [58]. The dashed green curve corresponds to the relativistic Boltzmann equation in the relaxation time approximation. The thermodynamic quantities in this model have been estimated using the scaled hadron masses and coupling (SHMC) model [96]. The brown dashed curve corresponds to estimations made using the relativistic Boltzmann equation in RTA. The thermodynamic quantities are estimated within the excluded volume hadron resonance gas model (EHRG) [95]. The dot-dashed orchid curve corresponds to the $\eta / s$ of meson gas estimated using chiral perturbation theory [76]. While the ratio $\eta / s$ in our model is relatively large at low temperature as compared to other models, it rapidly falls and approaches the Kovtun-Son-Starinets (KSS) bound at $T \sim 170 \mathrm{MeV}$.

Figure 5 shows the ratio of bulk viscosity to entropy density as a function of temperature. In Fig. 5(a) the blue solid curve corresponds to the RMFHRG compared with that of the EHRG model (dashed magenta curve) [95] and the SHMC model [96]. Note that the ratio $\zeta / s$ is smaller when the repulsive interactions are treated in a mean field way. Figure 5(a) shows the ratio $\zeta / s$ at finite baryon chemical potential. At low temperature the ratio is larger at finite $\mu$; it drops below that of the $\mu=0$ case at high temperature. This observation may be attributed to the fact that the entropy density rises much faster than that of $\zeta$ itself at finite baryon density as compared to that of zero baryon density case.

In the context of heavy nucleon-nucleon (NN) collision experiments, viscosity coefficients can be estimated along the freeze-out curve by finding the beam energy $\left(\sqrt{S_{N N}}\right)$ dependence of the temperature and chemical potential. 


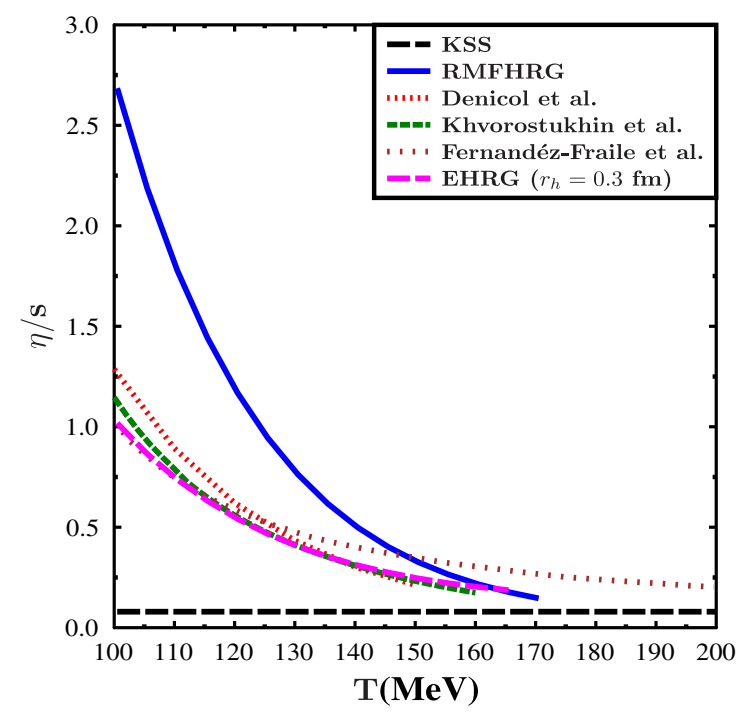

(a)

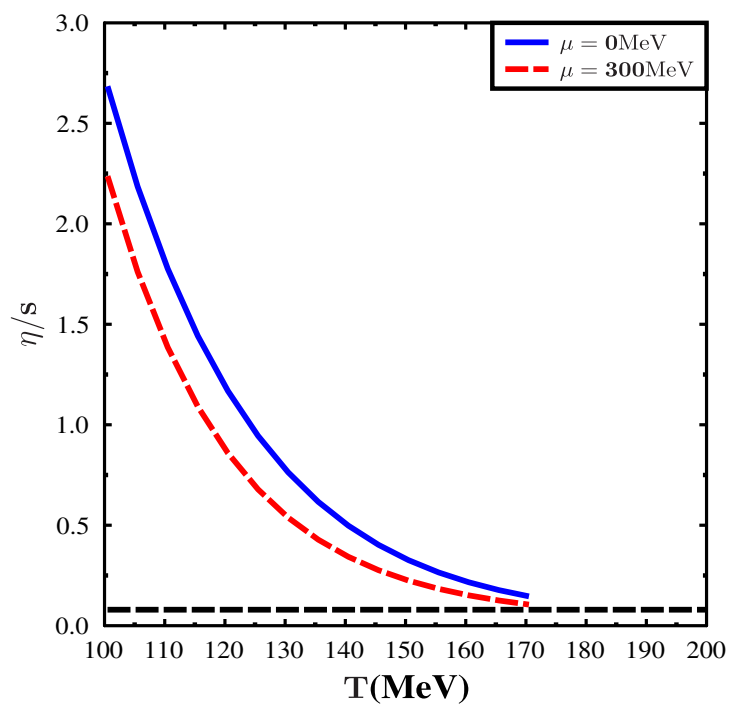

(b)

FIG. 4. The left panel shows the shear viscosity to entropy density ratio estimated within the RMFHRG and compared with other model estimations. These figures correspond to $\mu=0$. The right panel shows $\eta / s$ at two different baryon chemical potentials.

This is extracted from a statistical thermal model description of the particle yield at various $\sqrt{S_{N N}}$ [113-115]. We use the parametrization of the freeze-out curve $T\left(\mu_{B}\right)$ given in Ref. [114] as

$T\left(\sqrt{S_{N N}}\right)=c_{+}\left(T_{10}+T_{20} \sqrt{S_{N N}}\right)+c_{-}\left(T_{0}^{\lim }+\frac{T_{30}}{\sqrt{S_{N N}}}\right)$,

$$
\mu\left(\sqrt{S_{N N}}\right)=\frac{a_{0}}{1+b_{0} \sqrt{S_{N N}}}
$$

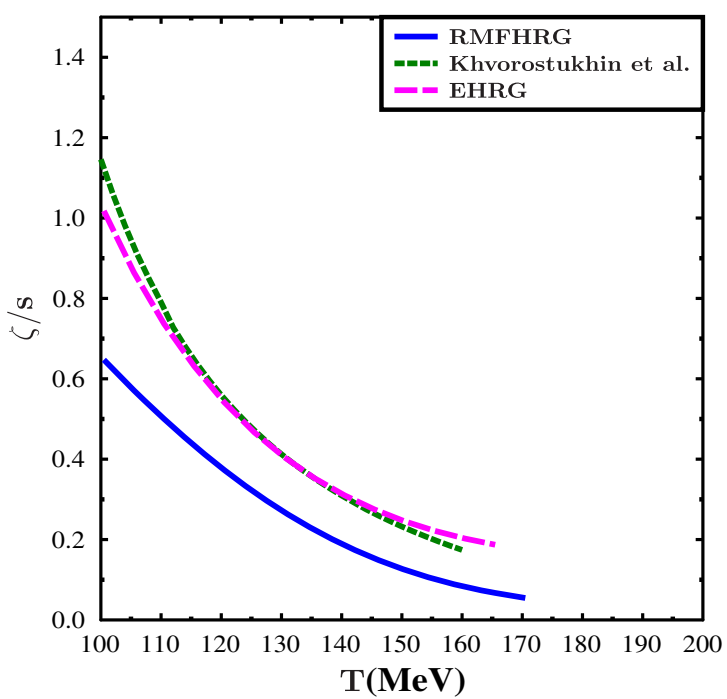

(a) where $T_{10}=-34.4 \mathrm{MeV}, T_{20}=30.9 \mathrm{MeV} / \mathrm{GeV}, T_{30}=$ $-176.8 \mathrm{GeV} \mathrm{MeV}, T_{0}^{\lim }=161.5 \mathrm{MeV}, a_{0}=1481.6 \mathrm{MeV}$ and $b_{0}=0.365 \mathrm{GeV}^{-1}$. The functions $c_{+}$and $c_{-}$ smoothly connect the different behaviors of center-of-mass energies.

Figure 6 shows viscosity coefficients $\eta / s$ and $\zeta / s$ along the freeze-out curve. It can be noted that the fluidity measure rapidly falls at low $\sqrt{S}$, and then it remains almost constant at higher $\sqrt{S}$ values. This indicates that the matter produced in heavy-ion collision experiments with a wide range of collision energies can exhibit substantial elliptic flow.

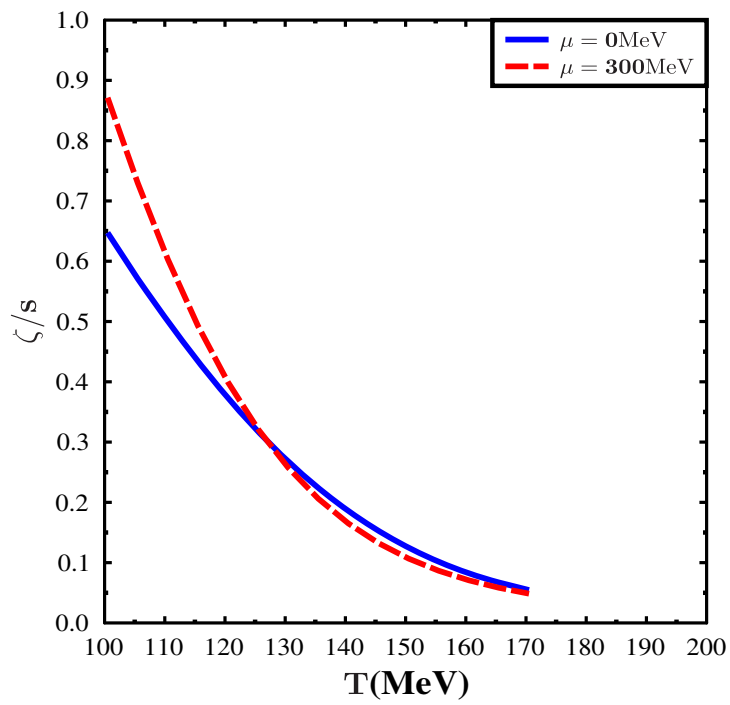

(b)

FIG. 5. The left panel shows the bulk viscosity to entropy density ratio estimated within the RMFHRG and compared with other model estimations. These results are for $\mu=0$. The right panel shows $\zeta / s$ at two different baryon chemical potentials. 


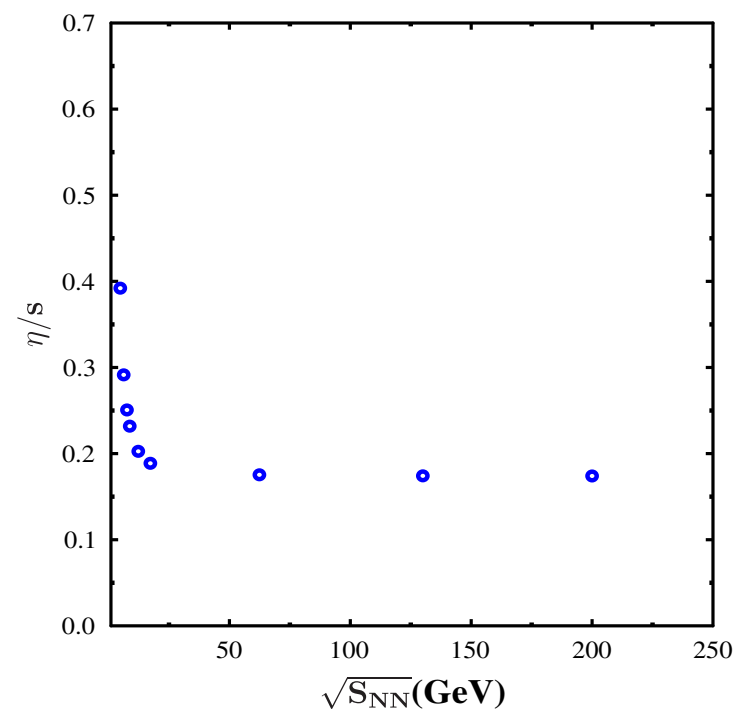

(a)

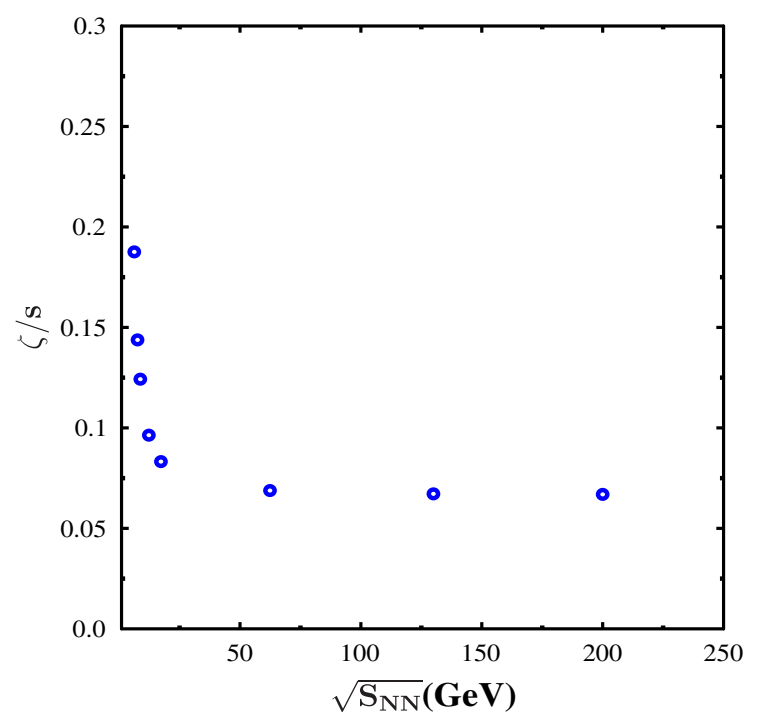

(b)

FIG. 6. Viscosity coefficients along the freeze-out curve. The freeze-out parametrization is taken from Ref. [114].

\section{SUMMARY}

In this paper we confronted the RMFHRG with LQCD at zero as well as finite density. The repulsive interaction between hadrons is treated using the mean field approach. The thermodynamic quantities estimated within the RMFHRG are found to be in reasonable agreement with LQCD at zero as well as finite chemical potential. The agreement of the interaction measure $\epsilon-3 P / T^{4}$ estimated within the RMFHRG is rather poor above $T=145 \mathrm{MeV}$. In fact, the interaction measure rises very rapidly near $T_{c} \sim 156 \mathrm{MeV}$. This rapid rise of energy density can be explained by extending the ideal HRG model by including continuum Hagedorn states along with the discrete states. We used this RMFHRG equation of state to estimate the shear and bulk viscosity coefficients of hadronic matter. We found reasonable agreement of both the viscosity coefficients with previous results. The shear viscosity to entropy density ratio $\eta / s$ estimated within the RMFHRG is large at low temperature as compared to other calculations. This behavior is due to the smaller cross section of mesons in our model. But $\eta / s$ estimated in our calculation rapidly drops at high temperature and approaches the KSS bound at $T \sim 170 \mathrm{MeV}$. We further found that $\eta / s$ at finite chemical potential is smaller in magnitude as compared to that of zero chemical potential, but the overall behavior as a function of temperature does not change. We also found reasonable agreement of the ratio $\zeta / s$ with previous results. Finally, we have estimated viscosity coefficients along the freeze-out line. We found that both the ratios $\eta / s$ and $\zeta / s$ attain constant values at high $\sqrt{S}$ values. This indicates that the matter produced in heavy-ion collision experiments with a wide range of collision energies can exhibit substantial elliptic flow.

\section{ACKNOWLEDGMENTS}

G. K. is financially supported by the DST-INSPIRE faculty award under Grant No. DST/INSPIRE/04/2017/ 002293.
[1] Y. Aoki, Z. Fodor, S. D. Katz, and K. K. Szabo, Phys. Lett. B 643, 46 (2006).

[2] S. Borsanyi, Z. Fodor, C. Hoelbling, S. D. Katz, S. Krieg, C. Ratti, and K. K. Szabó (Wuppertal-Budapest Collaboration), J. High Energy Phys. 09 (2010) 073.

[3] S. Borsanyi, C. Hoelbling, B. C. Toth, S. Durr, S. Krieg, K. K. Szabo, Z. Fodor, S. D. Katz, D. Nogradi, and N. Trombitas, Phys. Rev. D 92, 014505 (2015).

[4] P. Petreczky, AIP Conf. Proc. 1520, 103 (2013).
[5] H. T. Ding, F. Karsch, and S. Mukherjee, Int. J. Mod. Phys. E 24, 1530007 (2015).

[6] B. Friman, C. Hohne, J. Knoll, S. Leupold, J. Randrup, R. Rapp, and P. Senger, Lect. Notes Phys. 814, 11 (2011).

[7] A. Bazavov et al. (HotQCD Collaboration), Phys. Lett. B 795, 15 (2019).

[8] S. Borsanyi, G. Endrodi, Z. Fodor, S. D. Katz, S. Krieg, C. Ratti, and K. K. Szabo, J. High Energy Phys. 08 (2012) 053. 
[9] A. Bazavov et al., Phys. Rev. D 95, 054504 (2017).

[10] S. Datta, R. V. Gavai, and S. Gupta, Phys. Rev. D 95, 054512 (2017).

[11] S. Datta, R. V. Gavai, and S. Gupta, Proc. Sci., LATTICE2013 (2014) 202.

[12] S. P. Klevansky, Rev. Mod. Phys. 64, 649 (1992).

[13] T. Hatsuda and T. Kunihiro, Phys. Rep. 247, 221 (1994).

[14] B. J. Schaefer, J. M. Pawlowski, and J. Wambach, Phys. Rev. D 76, 074023 (2007).

[15] R. Dashen, S. K. Ma, and H. J. Bernstein, Phys. Rev. 187, 345 (1969).

[16] R. F. Dashen and R. Rajaraman, Phys. Rev. D 10, 694 (1974).

[17] G. M. Welke, R. Venugopalan, and M. Prakash, Phys. Lett. B 245, 137 (1990).

[18] R. Venugopalan and M. Prakash, Nucl. Phys. A546, 718 (1992).

[19] P. Braun-Munzinger, J. Stachel, J. P. Wessels, and N. Xu, Phys. Lett. B 365, 1 (1996).

[20] G. D. Yen and M. I. Gorenstein, Phys. Rev. C 59, 2788 (1999).

[21] F. Becattini, J. Cleymans, A. Keranen, E. Suhonen, and K. Redlich, Phys. Rev. C 64, 024901 (2001).

[22] J. Cleymans and H. Satz, Z. Phys. C 57, 135 (1993).

[23] P. Braun-Munzinger, D. Magestro, K. Redlich, and J. Stachel, Phys. Lett. B 518, 41 (2001).

[24] J. Rafelski and J. Letessier, Nucl. Phys. A715, 98 (2003).

[25] A. Andronic, P. Braun-Munzinger, and J. Stachel, Nucl. Phys. A772, 167 (2006).

[26] S. Chatterjee, R. M. Godbole, and S. Gupta, Phys. Lett. B 727, 554 (2013).

[27] S. Chatterjee, S. Das, L. Kumar, D. Mishra, B. Mohanty, R. Sahoo, and N. Sharma, Adv. High Energy Phys. (2015) 349013.

[28] M. Albright, J. Kapusta, and C. Young, Phys. Rev. C 92 , 044904 (2015).

[29] V. Vovchenko, M. I. Gorenstein, and H. Stoecker, Phys. Rev. Lett. 118, 182301 (2017).

[30] V. Vovchenko, A. Motornenko, P. Alba, M. I. Gorenstein, L. M. Satarov, and H. Stoecker, Phys. Rev. C 96, 045202 (2017).

[31] P. Alba, W. M. Alberico, A. Nada, M. Panero, and H. Stöcker, Phys. Rev. D 95, 094511 (2017).

[32] P. Braun-Munzinger, I. Heppe, and J. Stachel, Phys. Lett. B 465, 15 (1999).

[33] D. H. Rischke, J. Schaffner, M. I. Gorenstein, A. Schaefer, H. Stoecker, and W. Greiner, Z. Phys. C 56, 325 (1992).

[34] C. P. Singh, B. K. Patra, and K. K. Singh, Phys. Lett. B 387, 680 (1996).

[35] J. I. Kapusta and K. A. Olive, Nucl. Phys. A408, 478 (1983).

[36] K. A. Olive, Nucl. Phys. B190, 483 (1981).

[37] P. Huovinen and P. Petreczky, Phys. Lett. B 777, 125 (2018).

[38] C. Gale, S. Jeon, and B. Schenke, Int. J. Mod. Phys. A 28, 1340011 (2013).

[39] B. Schenke, J. Phys. G 38, 124009 (2011).

[40] C. Shen and U. Heinz, Phys. Rev. C 85, 054902 (2012).

[41] P. F. Kolb and U. W. Heinz, arXiv:nucl-th/0305084.
[42] D. Teaney, J. Lauret, and E. V. Shuryak, Phys. Rev. Lett. 86, 4783 (2001).

[43] L. Del Zanna, V. Chandra, G. Inghirami, V. Rolando, A. Beraudo, A. De Pace, G. Pagliara, A. Drago, and F. Becattini, Eur. Phys. J. C 73, 2524 (2013).

[44] I. Karpenko, P. Huovinen, and M. Bleicher, Comput. Phys. Commun. 185, 3016 (2014).

[45] H. Holopainen, H. Niemi, and K. J. Eskola, J. Phys. G 38, 124164 (2011).

[46] A. Jaiswal, B. Friman, and K. Redlich, Phys. Lett. B 751, 548 (2015).

[47] M. Gyulassy and L. McLerran, Nucl. Phys. A750, 30 (2005).

[48] L. P. Csernai, J. I. Kapusta, and L. D. McLerran, Phys. Rev. Lett. 97, 152303 (2006).

[49] P. Kovtun, D. T. Son, and A. O. Starinets, Phys. Rev. Lett. 94, 111601 (2005).

[50] S. Gavin, Nucl. Phys. A435, 826 (1985).

[51] A. Hosoya and K. Kajantie, Nucl. Phys. B250, 666 (1985).

[52] M. Prakash, M. Prakash, R. Venugopalan, and G. Welke, Phys. Rep. 227, 321 (1993).

[53] A. Dobado and F. J. Llanes-Estrada, Phys. Rev. D 69, 116004 (2004).

[54] J. W. Chen, Y. H. Li, Y. F. Liu, and E. Nakano, Phys. Rev. D 76, 114011 (2007).

[55] K. Itakura, O. Morimatsu, and H. Otomo, Phys. Rev. D 77, 014014 (2008).

[56] A. Dobado, F. J. Llanes-Estrada, and J. M. Torres-Rincon, Phys. Rev. D 80, 114015 (2009).

[57] N. Demir and S. A. Bass, Phys. Rev. Lett. 102, 172302 (2009).

[58] G. S. Denicol, C. Gale, S. Jeon, and J. Noronha, Phys. Rev. C 88, 064901 (2013).

[59] A. Puglisi, S. Plumari, and V. Greco, Phys. Lett. B 751, 326 (2015).

[60] L. Thakur, P. K. Srivastava, G. P. Kadam, M. George, and H. Mishra, Phys. Rev. D 95, 096009 (2017).

[61] G. Kadam, S. Pawar, and H. Mishra, J. Phys. G 46, 015102 (2019).

[62] G. Kadam and S. Pawar, Adv. High Energy Phys. (2019) 6795041.

[63] M. I. Gorenstein, M. Hauer, and O. N. Moroz, Phys. Rev. C 77, 024911 (2008).

[64] J. Noronha-Hostler, J. Noronha, and C. Greiner, Phys. Rev. C 86, 024913 (2012).

[65] K. Rajagopal and N. Tripuraneni, J. High Energy Phys. 03 (2010) 018.

[66] J. R. Bhatt, H. Mishra, and V. Sreekanth, Phys. Lett. B 704, 486 (2011); Nucl. Phys. A875, 181 (2012); Phys. Rev. C 80, 054906 (2009).

[67] A. Monnai and T. Hirano, Phys. Rev. C 80, 054906 (2009).

[68] G. S. Denicol, T. Kodama, T. Koide, and P. Mota, Phys. Rev. C 80, 064901 (2009).

[69] K. Dusling and T. Schäfer, Phys. Rev. C 85, 044909 (2012).

[70] H. Song and U. W. Heinz, Phys. Rev. C 81, 024905 (2010).

[71] J. Noronha-Hostler, G. S. Denicol, J. Noronha, R. P. G. Andrade, and F. Grassi, Phys. Rev. C 88, 044916 (2013).

[72] J. Noronha-Hostler, J. Noronha, and F. Grassi, Phys. Rev. C 90, 034907 (2014). 
[73] A. Dobado and S. N. Santalla, Phys. Rev. D 65, 096011 (2002).

[74] D. Davesne, Phys. Rev. C 53, 3069 (1996).

[75] D. Kharzeev and K. Tuchin, J. High Energy Phys. 09 (2008) 093.

[76] D. Fernandez-Fraile and A. Gomez Nicola, Eur. Phys. J. C 62, 37 (2009).

[77] J. W. Chen and J. Wang, Phys. Rev. C 79, 044913 (2009).

[78] J. Noronha-Hostler, J. Noronha, and C. Greiner, Phys. Rev. Lett. 103, 172302 (2009); Phys. Rev. C 86, 024913 (2012).

[79] C. Sasaki and K. Redlich, Nucl. Phys. A832, 62 (2010); Phys. Rev. C 79, 055207 (2009).

[80] D. Fernandez-Fraile and A. Gomez Nicola, Phys. Rev. Lett. 102, 121601 (2009).

[81] A. Dobado and J. M. Torres-Rincon, Phys. Rev. D 86, 074021 (2012).

[82] V. Ozvenchuk, O. Linnyk, M. I. Gorenstein, E. L. Bratkovskaya, and W. Cassing, Phys. Rev. C 87, 064903 (2013).

[83] U. Gangopadhyaya, S. Ghosh, S. Sarkar, and S. Mitra, Phys. Rev. C 94, 044914 (2016).

[84] P. Chakraborty and J. I. Kapusta, Phys. Rev. C 83, 014906 (2011).

[85] C. Sasaki and K. Redlich, Phys. Rev. C 79, 055207 (2009).

[86] H. Berrehrah, E. Bratkovskaya, W. Cassing, and R. Marty, J. Phys. Conf. Ser. 612, 012050 (2015).

[87] R. Marty, E. Bratkovskaya, W. Cassing, J. Aichelin, and H. Berrehrah, Phys. Rev. C 88, 045204 (2013).

[88] S. Samanta, S. Ghosh, and B. Mohanty, J. Phys. G 45, 075101 (2018).

[89] K. Saha, S. Ghosh, S. Upadhaya, and S. Maity, Phys. Rev. D 97, 116020 (2018).

[90] P. Singha, A. Abhishek, G. Kadam, S. Ghosh, and H. Mishra, J. Phys. G 46, 015201 (2019).

[91] D. Fernandez-Fraile and A. Gomez Nicola, Int. J. Mod. Phys. E 16, 3010 (2007).

[92] S. Sarkar, Adv. High Energy Phys. (2013) 627137.

[93] P. Deb, G. P. Kadam, and H. Mishra, Phys. Rev. D 94, 094002 (2016).

[94] A. Abhishek, H. Mishra, and S. Ghosh, Phys. Rev. D 97, 014005 (2018).
[95] G. P. Kadam and H. Mishra, Phys. Rev. C 92, 035203 (2015).

[96] A. S. Khvorostukhin, V. D. Toneev, and D. N. Voskresensky, Nucl. Phys. A845, 106 (2010).

[97] A. Dash, S. Samanta, and B. Mohanty, Phys. Rev. D 100, 014025 (2019).

[98] H. X. Zhang, J. W. Kang, and B. W. Zhang, arXiv:1905 .08146 .

[99] V. Mykhaylova, M. Bluhm, K. Redlich, and C. Sasaki, Phys. Rev. D 100, 034002 (2019).

[100] C. A. Islam, J. Dey, and S. Ghosh, arXiv:1901.09543.

[101] S. Ghosh, F. E. Serna, A. Abhishek, G. Krein, and H. Mishra, Phys. Rev. D 99, 014004 (2019).

[102] S. Ghosh, S. Ghosh, and S. Bhattacharyya, Phys. Rev. C 98, 045202 (2018).

[103] F. Gao and Y. x. Liu, Phys. Rev. D 97, 056011 (2018).

[104] S. Ghosh, S. Chatterjee, and B. Mohanty, Phys. Rev. C 94, 045208 (2016).

[105] M. Attems, J. Casalderrey-Solana, D. Mateos, D. SantosOliván, C. F. Sopuerta, M. Triana, and M. Zilhão, J. High Energy Phys. 01 (2017) 026.

[106] M. Attems, J. Casalderrey-Solana, D. Mateos, D. SantosOliván, C. F. Sopuerta, M. Triana, and M. Zilhão, J. High Energy Phys. 06 (2017) 154.

[107] W. Florkowski, E. Maksymiuk, and R. Ryblewski, Phys. Rev. C 97, 024915 (2018).

[108] M. Tanabashi et al. (Particle Data Group), Phys. Rev. D 98, 030001 (2018).

[109] M. Albright and J. Kapusta, Phys. Rev. C 93, 014903 (2016).

[110] David J. Griffiths, Introduction to Quantum Mechanics, 2nd ed. (Pearson Education, Singapore, 2005).

[111] M. Cannoni, Phys. Rev. D 89, 103533 (2014).

[112] P. Gondolo and G. Gelmini, Nucl. Phys. B360, 145 (1991).

[113] J. Cleymans, H. Oeschler, K. Redlich, and S. Wheaton, Phys. Rev. C 73, 034905 (2006).

[114] K. A. Bugaev, A. I. Ivanytskyi, D. R. Oliinychenko, E. G. Nikonov, V. V. Sagun, and G. M. Zinovjev, Ukr. J. Phys. 60, 181 (2015).

[115] A. Tawfik, Nucl. Phys. A922, 225 (2014). 